\title{
Gut Microbiome Analysis Reveals Characteristics Decrease in Bifidobacterium Count in the Adult Type 2 Diabetes Mellitus Patients
}

\section{Muzammal Sattar}

Government College University Faisalabad

Haseeb Anwar ( $\boldsymbol{\sim}$ drhaseebanwar@gcuf.edu.pk)

Government College University Faisalabad https://orcid.org/0000-0001-8438-9700

\section{Muhammad Umar Sohail}

QBRI: Qatar Biomedical Research Institute

\author{
Irshad Ahmad
}

MoPH: Ministry of Public Health Qatar

\section{Muhamm Naeem Faisal}

University of Agriculture Faisalabad

\section{Zahid Kamran}

Islamia University of Bahawalpur: The Islamia University of Bahawalpur Pakistan

\section{Maha Al Asmakh \\ Qatar University}

\section{Research}

Keywords: Diabetes, Microbiome, Bifidobacterium, Leptin, Amylin

Posted Date: October 13th, 2020

DOl: https://doi.org/10.21203/rs.3.rs-90715/v1

License: (c) (i) This work is licensed under a Creative Commons Attribution 4.0 International License. Read Full License 


\section{Abstract}

Background: Type 2 diabetes mellitus is an idiopathic metabolic disorder in epidemic phase. The current study examines the gut microbiome of diabetic (Diab), diabetic with secondary complications (DSC), and healthy control (HC) subjects enrolled at the district hospital, Faisalabad, Pakistan. The study groups were characterized based on serum glucose and insulin concentrations and the subject's clinical history. Serum IgM, leptin, and amylin concentrations were determined using ELISA. The stool microbiome was analyzed using 16S rRNA amplicon sequencing on the Illumina MiSeq.

Results: Serum IgM, leptin, and amylin concentrations were higher $(p \leq 0.05)$ in the Diab and DSC groups compared with the HC group. Results: No statistical differences were observed in microbiome composition and diversity at community levels as evaluated by alpha (Kruskal-Wallis $p=0.16$ ) and beta (PERMANOVA $p=0.41$ ) diversity indexes. Firmicutes $(51.2 \%)$, Proteobacteria $(14.9 \%)$, Actinobacteria (12.5\%), and Bacteroidetes (7.8\%) were the predominant bacterial phyla present in the stool microbiome of the study population. However, no differences $(p \geq 0.05)$ in phylum-level taxonomic composition were observed between the study groups. At genus level, Coprococcus and Ruminococcus from phylum Firmicutes and Bifidobacterium from phylum Actinobacteria were significantly different $(p \leq 0.05)$ between the study groups. Bifidobacterium and Coprococcus were higher in the HC group, whereas Ruminococcus was higher in the DSC group.

Conclusions: Taken together, in the present study, we report the gut microbiome of diabetic patients, which has specific taxonomic characteristics associated with the incidence and progression of diabetes.

\section{Background}

Diabetes mellitus is an idiopathic metabolic disease with severe secondary complications and a life-time disability. In recent years, the global prevalence of the disease has reached an epidemic phase at an alarming pace [1]. The progression of hyperglycemia into type 2 diabetes mellitus (T2DM) in susceptible adults is contributed by genetic and environmental factors [2]. In general, gut microbes exist in a state of symbiosis with their host. However, environmental factors, such as diet and lifestyle habits, may perturb this symbiotic association and lead to unfavorable health consequences [3, 4]. The significance of the gut microbiome in diabetes pathogenesis remains elusive. Although, recently emerged notion of shared genetic material and functional dogma between host and its microbiome highlights the dominant role of the microbiome in the development of T2DM and other obesity-related metabolic diseases [5].

Recently, the gut microbiome has become a key area of research as its manipulation could potentially help to manage several diseases, including obesity and diabetes [6]. Microbial dysbiosis, characterized by a decline in bacterial richness and diversity, may enhance intestinal permeability, glucose homeostasis, and elicit systemic inflammation [7]. Previous diabetes-microbiome studies demonstrate several vital changes in gut microbiome composition, including a decline in microbial diversity and relative richness of specific microbial taxa at different hierarchical levels $[5,6,8-10]$. Literature review also reveals a rise in 
Firmicutes to Bacteroidetes ratio (F/B) in the subjects with T2DM compared to the healthy controls. Furthermore, the F/B has been positively associated with serum glucose, whereas, Bifidobacterium and Roseburia are positively and negatively associated with serum glucose, respectively $[8,10]$. Specifically, Bifidobacterium [10], Coprococcus [11], and Ruminococcus [11, 12] have been involved in energy homeostasis and may be associated in the transition from normoglycemia to pre-diabetes and T2DM [13].

Supporting a causal role of gut microbiome in diabetes, human fecal transplantation studies show improvement in insulin sensitivity in recipients with metabolic syndrome [14]. However, the precise mechanism through which the microbiome contributes to the T2DM is not fully understood. Furthermore, many diabetes-microbiome studies have produced inconsistent observations about the explicit nature of microbiome associations with T2DM. The data from three large cohort studies [15-17]; conducted in America, China, and Europe, suggest a fundamental role of gender and ethnicity in the gut microbial composition, indicating an ambiguity in generalizing diabetes-microbiome association, particularly to ethnic groups underrepresented in research studies and clinical trials. Therefore, to address the critical knowledge gap, we examine the gut microbiome of T2DM patients attending District Headquarter Hospital Faisalabad, Pakistan. We analyze the association of the gut microbiome with serum leptin, amylin, and IgM levels. To the best of our knowledge, this is the first diabetes-microbiome study from Pakistan, a country with an estimated $14.62 \%$ prevalence of T2DM [18].

\section{Methods}

\subsection{Study cohort}

This cross-sectional study enrolled twenty-two male adults (age group 40 to 60 years) volunteers from district headquarter hospital, Faisalabad, Pakistan. All participants were divided into three groups; healthy control ( $\mathrm{HC} ; \mathrm{N}=6$ ), type 2 diabetes (Diab; $N=9$ ), and Diab subjects with secondary complications (DSC; $N$ $=7$ ). The inclusion criteria for the participants were: age $\geq 30$ years, in good general health $(\mathrm{HC})$, or diagnosed with diabetes type 2 with or without secondary complications. Individuals taking antibiotics in the preceding three months or unwilling to provide stool samples were excluded from the study. All the participants were informed about the study objects and protocols. Before the start of sampling, written formal consent was obtained from each participant. The institutional review board of the university approved this study in compliance with participant anonymity, research ethical, moral, and biosafety standards (Reference no. GCUF/ECR/13).

A diabetes study participation questionnaire was designed according to WHO standards to identify the participant's eligibility for the current study. Diab and DSC status was established by the patient's selfreported history. Furthermore, the diabetic status of the patients was confirmed by measuring random blood glucose and insulin concentrations. Information on disease history, secondary complications, and medication were collected from the participants. All study participants from the Diab and DSC groups were on diabetes medication. The selection of patients for the DSC group was based on their proven 
medical history in the hospital and their clinical profile. Mild to moderate nephropathy and cardiovascular diseases were the reported secondary complications in the DSC patients.

\subsection{Samples collection}

Blood samples were collected in a serum collection tube without clot activator. The serum was harvested by centrifugation at $1500 \times \mathrm{g}$ for 30 minutes.

A stool collection kit comprised of a sterile tube, spatula, gloves, and collection paper was provided to the participants. Stool samples were self-collected at the house by the participants and transported on ice to the hospital. All samples were preserved at $-20^{\circ} \mathrm{C}$ before DNA extraction.

\subsection{Serology biochemical and hormones analysis}

Serum insulin, leptin, amylase, and IgM antibodies concentrations were determined using commercially available kits. In brief, serum IgM (E-EL-H1814), insulin (E-EL-H2665), leptin (E-EL-HO113), and amylin (EEL-HO322) concentrations were measured using human ELISA kits (Elabscience Biotechnology Inc., Wuhan, China). The optical density of these reactions was measured spectrophotometrically at a wavelength of $450 \mathrm{~nm}$. The glucose (monoreagent- K082) concentration was measured by an enzymatic reaction following commercial kit protocol (Bioclin Systems II®, Quisaba, Bioclin, Belo Horizonte, MG, Brazil). The optical density was measured spectrophotometrically at a wavelength of $505 \mathrm{~nm}$.

\subsection{Stool microbiome analysis}

All stool samples were thawed on ice and weighed for further processing. Approximately 200 to $300 \mathrm{mg}$ of samples were used for DNA extraction using a commercially available kit [19]. In brief, the stool samples were re-suspended in $300 \mu \mathrm{L}$ of lysis solution containing lysozyme $(20 \mathrm{mg} / \mathrm{mL})$ and $100 \mathrm{X}$ Triton $(1.2 \%)$ in $1 \mathrm{X} \mathrm{TE}$ buffer. The samples were vigorously vortexed and incubated at $37^{\circ} \mathrm{C}$ for one hour. The quality and concentration of the genomic DNA was examined using NanoDrop-2000 (Thermo Fisher, US).

Purified DNA samples were subjected to amplicon sequencing library preparation protocol, as elaborated previously [20]. In brief, the DNA samples were subjected to PCR amplification using 337F/805R primerpair and index-primers. All libraries were washed and pooled before loading them in the Illumina MiSeq cartridge (MS-102-3003; Illumina, San Diego, CA, USA) [21]. Sequencing was performed on the lllumina MiSeq instrument at Applied Biological Materials Inc. Richmond, Canada.

\subsection{Bioinformatics analysis}

QIIME2 Bioinformatics pipeline was used for quality filtration, annotation, assembly, and FeatureTable construction, as previously described. ${ }^{8}$ In brief, FASTX toolkit was used for removing low-quality reads [22]. Paired-end reads were reassembled using DADA2 plugin to attain maximum overlap length [23]. Taxonomy classification was performed using VSEARCH, and BLAST + tools wrapped in q2-featureclassifier plugin.[24] Greengenes (gg_13_8) was used as a reference database for taxonomy classification. 


\subsection{Statistics analysis}

Only those taxa that had a relative abundance of at least $0.5 \%$ in all the three groups were included in statistical analysis, and remaining data were discarded. All data were presented as mean and statistical significance $(P \leq 0.05)$ between the groups was determined by one-way ANOVA, followed by Tukey's posthoc test. The Benjamini-Hochberg false discovery rate (FDR) was used to perform multiple comparisons of the P-values for the microbiome data, and an adjusted $\mathrm{P} \leq 0.05$ was considered statistically significant. Alpha- and beta-diversity analysis was performed at 15,653 sequencing depth and differences between the groups were observed using theKruskal-Wallis and PERMANOVA test, respectively.

\section{Results}

The mean serum glucose concentration was higher $(p \leq 0.05)$ in the Diab and DSC groups as compared with the $\mathrm{HC}$ group (data not presented).Concordantly, the serum insulin concentration was lower ( $\mathrm{p} \leq$ 0.05 ) in the Diab and DSC groups as compared to the HC group. Serum IgM concentration was higher ( $p$ $\leq 0.05$ ) in the Diab group compared with the HC and DSC groups. Serum leptin and amylin concentrations were also higher $(\mathrm{p} \leq 0.05)$ in the Diab group compared with the HC group. However, the differences between the DSC group and the Diab and HC groups were statistically non-significant (Fig. 1).

Illumina MiSeq sequencing produced 2,243,433 sequences for all the analyzed samples $(n=22$, median \pm $S D=91,821 \pm 54,999)$. After quality filtration and chimera removal, 438,285 sequences of good quality (Phred quality score $\geq$ ASCII 30), belonging to 3,585 features (OTUs), were used for further analysis. These sequences correspond to 50 phyla, 149 classes, 253 orders, 362 families, 522 genera, and 583 species. In descending order, the four most dominant phyla present in the stool microbiome of the study population were Firmicutes (51.2\%), Proteobacteria (14.9\%), Actinobacteria (12.5\%), and Bacteroidetes (7.8\%) (Fig. 2). Among these abundantly present phyla, Actinobacteria, Proteobacteria, and Firmicutes to Bacteroidetes were numerically different among the groups. Similarly, at family and genus levels, Bifidobacteriaceae $(p=0.03)$ and Bifidobacterium $(p=0.02)$ were more abundantly present in the HC group compared with the Diab and DSC groups. Discordantly, family Ruminococcaceae $(p \leq 0.05)$ and genus Ruminococcus $(p \leq 0.05)$ were more abundant in the DSC group compared with the Diab and HC groups (Fig. 3). However, when Benjamini-Hochberg FDR was applied to these statistically different clades, their significance was lost, and only family Bifidobacteriaceae and genus Bifidobacterium were left statistically different $(p \leq 0.05)$.

Figure 4a depicts the alpha-diversity index of bacterial communities in the three study groups. The observed_OTUs index was utilized to determine taxonomic diversity (species richness) within the samples. However, no significant differences were observed between the HC, Diab, and DSC groups (Kruskal-Wallis $p \geq 0.05$ ). Similarly, beta-diversity was also non-significantly different between the study groups. The weighted_unifrac distance matrix-based PCoA plot showed that no distinct clustering pattern was present between the microbiome of the study samples (Fig. 4b). The Permutational multivariate 
analysis of variance (PERMANOVA) tests applied in the beta-diversity index did not show significant differences (PERMANOVA $p=0.41$ ) among the studied groups.

\section{Discussion}

In the present study, we analyzed serum leptin, amylin, and IgM concentrations and stool microbiome of the healthy, diabetic, and diabetic with secondary complications subjects. The microbiome data were presented at phylum, family, and genus levels. Furthermore, the microbiome was also analyzed at the community level using alpha- and beta-diversity methods to classify specific community features that differed between the three studied groups.

In this study, we found that higher serum leptin and amylin levels were associated with diabetes mellitus. Amylin, a peptide hormone, plays an important role in pancreatic beta-cell damage in T2DM patients through islet amyloid formation and progression of secondary cardiovascular and nephropathies [25, 26]. In diabetes patients, theco-secretion of amylin and insulin from beta-cells in response to the glucose decreases [27]. Similarly, leptin secretion is closely related to body fat contents, and it is suggested that serum leptin may be a good biomarker of obesity-associated T2DM.[28] Higher leptin levels in obese subjects may promote the development of T2DM and secondary metabolic complications [28, 29]. Therefore, both amylin and leptin are associated with either loss of beta-cell mass or impaired insulin sensitivity on peripheral tissues in T2DM patients [30]. However, previous studies examine the association between serum leptin concentration and T2DM were not consistent in their findings. Few studies described a significantly positive association between serum leptin and the development of T2DM [31, 32], while others reported positive associations in males only [33, 34] or even negative association $[35,36]$. It is possible that these inconsistencies in previous findings might be due to differences in sampling techniques or the subject's inclusion and exclusion criteria.

The gastrointestinal tract microbiome was investigated using 16S rRNA amplicon sequencing, and the data were presented at different taxonomic levels. Comparisons between community composition and taxonomic diversity were made between the study groups. Here we observed that microbial community diversity, as revealed by alpha- and beta-diversity indexes were not significantly different between the study groups. These observations resemble with our previous findings [9], where we report no statistical differences between the salivary microbiome diversity of obese pre-diabetic and healthy lean controls. Contrary to our observations, many researchers hypothesize that the diabetic phenotype is associated with a decline in gut microbial diversity, characterized as species richness and abundance $[8,17,37]$. These studies concluded that diverse gut microbiome could have a protective effect against systemic inflammation and metabolic disorders.

Similarly, no difference was observed between Firmicutes to Bacteroidetes ratio in the study groups, another critical parameter, most often emphasized as a prodrome for metabolic disorders development [9]. However, no significant differences in quantifications of taxonomic composition at phylum were observed in this study among the study groups. In the current study, only a few interesting observations 
were reported at the family and genus level; particularly, a decline in population densities of the Bifidobacteriaceae and Bifidobacterium in the diabetic subjects. Previous diabetes-microbiome studies also correlate genus Bifidobacterium from the phylum Actinobacteria with a decreased risk of T2DM [10, 38, 39]. In general, the members of family Bifidobacteriaceae are known for lowering metabolic endotoxemia and glucose intolerance [10]. Previous studies show that increase in Bifidobacterium level improved glucose-tolerance, insulin secretion, and sensitivity and reduced systemic endotoxemia through enhanced gut barrier function.[40,41] Particularly, probiotic and prebiotic supplementations in T2DM adults that reported increase in Bifidobacterium and Coprococcus counts also showed marked improvement in glucose and lipid homeostasis [42]. Besides, restoration of Bifidobacterium and Coprococcus spp., these supplements were also associated with alleviations of hyperglycemia, hyperlipidemia, and hepatic steatosis in mice fed with HFD $[43,44]$.

\section{Conclusion}

In conclusion, we observed that subjects with diabetic and diabetic secondary complication have different serum biochemical and the gut microbiome profiles. Results of this study reveal a bacterial dysbiosis in stool samples of disease subjects with a significant drop in the concentrations of family Bifidobacteriaceae, which may be considered as T2DM confounding factors. These findings suggest that gut microbiome's effects on T2DM would not be mediated through microbiome composition but rather through its function contents as closely related taxa may have widely different functions while distantly related taxa can have similar features.

\section{Declarations}

\section{Ethics approval and consent to participate}

The study was proved from institutional review board of the Government College University, Faisalabad (Reference no. GCUF/ECR/13).

\section{Consent for publication}

All the authors have read the draft before submission and have agreed with the contents.

\section{Availability of data and material}

The raw sequencing data in the form of FASTQ is being submitted to NCBI-SRA. Once obtained, the project ID will be mentioned in the draft.

\section{Competing interests}

All the authors declare that they do not have any competing interests with the submitted results.

\section{Funding}


This study was funded by Department of Physiology, GC University Faisalabad research fund. MZ is PhD Scholar at Department of Physiology. Microbiome sequencing was sponsored by Qatar University Internal Grant QUCP-CHS-2019-1.

\section{Authors' Contributions}

MS collected samples and perform laboratory procedures. MUS conceived the concept and performed bioinformatics analysis. HA supervised the laboratory work and contributed to writing the manuscript. MNF, IA, and ZK contributed to Statistical analysis, paper draft and technical review. MA performs statistical analysis and contributed to writing the manuscript.

\section{Acknowledgment}

The authors acknowledge Dr Imran Mukhtar and Mr Imtiaz Mustafa for their help in laboratory analysis and samples collection.

\section{References}

1. Cho N, Shaw J, Karuranga S, Huang Y, da Rocha Fernandes J, Ohlrogge A, Malanda B: IDF Diabetes Atlas: Global estimates of diabetes prevalence for 2017 and projections for 2045. Diabetes research and clinical practice 2018, 138:271-281.

2. Basit A, Fawwad A, Qureshi H, Shera A: Prevalence of diabetes, prediabetes and associated risk factors: second National Diabetes Survey of Pakistan (NDSP), 2016-2017. BMJ open 2018, 8:e020961.

3. Anwar H, Suchodolski JS, Ullah MI, Hussain G, Shabbir MZ, Mustafa I, Sohail MU: Shiitake CulinaryMedicinal Mushroom, Lentinus edodes (Agaricomycetes), Supplementation Alters Gut Microbiome and Corrects Dyslipidemia in Rats. International journal of medicinal mushrooms 2019, 21.

4. Asad F, Anwar H, Yassine H, Ullah MI, ul Rahman A, Kamran Z, Sohail MU: Agaricus bisporus mushroom and a probiotics mixture supplementation correct dyslipidemia without influencing the colon microbiome profile in hypercholesterolemic rats.

5. Liu Y, Wang Y, Ni Y, Cheung CK, Lam KS, Wang Y, Xia Z, Ye D, Guo J, Tse MA: Gut Microbiome Fermentation Determines the Efficacy of Exercise for Diabetes Prevention. Cell Metabolism 2020, 31:77-91. e75.

6. Sohail MU, Shabbir MZ, Steiner JM, Ahmad S, Kamran Z, Anwar H, Hussain G, Shaukat A, Suchodolski JS: Molecular analysis of the gut microbiome of diabetic rats supplemented with prebiotic, probiotic, and synbiotic foods. International Journal of Diabetes in Developing Countries 2017, 37:419-425.

7. Gurung M, Li Z, You H, Rodrigues R, Jump DB, Morgun A, Shulzhenko N: Role of gut microbiota in type 2 diabetes pathophysiology. EBioMedicine 2020, 51:102590. 
8. Larsen N, Vogensen FK, Van Den Berg FW, Nielsen DS, Andreasen AS, Pedersen BK, Al-Soud WA, Sørensen SJ, Hansen LH, Jakobsen M: Gut microbiota in human adults with type 2 diabetes differs from non-diabetic adults. PloS one 2010, 5.

9. Sohail MU, Yassine HM, Sohail A, Al Thani AA: Impact of Physical Exercise on Gut Microbiome, Inflammation, and the Pathobiology of Metabolic Disorders. Rev Diabet Stud 2019, 15:35-48.

10. Wu X, Ma C, Han L, Nawaz M, Gao F, Zhang X, Yu P, Li L, Zhou A, Wang J: Molecular characterisation of the faecal microbiota in patients with type II diabetes. Current microbiology 2010, 61:69-78.

11. Gomez-Arango LF, Barrett HL, Mclntyre HD, Callaway LK, Morrison M, Nitert MD: Connections between the gut microbiome and metabolic hormones in early pregnancy in overweight and obese women. Diabetes 2016, 65:2214-2223.

12. Remely M, Dworzak S, Hippe B, Zwielehner J, Aumüller E, Brath H, Haslberger A: Abundance and diversity of microbiota in type 2 diabetes and obesity. $J$ Diabetes Metab 2013, 4:2.

13. Zhang X, Shen D, Fang Z, Jie Z, Qiu X, Zhang C, Chen Y, Ji L: Human gut microbiota changes reveal the progression of glucose intolerance. PloS one 2013, 8.

14. Vrieze A, Van Nood E, Holleman F, Salojärvi J, Kootte RS, Bartelsman JF, Dallinga-Thie GM, Ackermans MT, Serlie MJ, Oozeer R: Transfer of intestinal microbiota from lean donors increases insulin sensitivity in individuals with metabolic syndrome. Gastroenterology 2012, 143:913-916. e917.

15. Ciubotaru I, Green SJ, Kukreja S, Barengolts E: Significant differences in fecal microbiota are associated with various stages of glucose tolerance in African American male veterans. Translational Research 2015, 166:401-411.

16. Karlsson FH, Tremaroli V, Nookaew I, Bergström G, Behre CJ, Fagerberg B, Nielsen J, Bäckhed F: Gut metagenome in European women with normal, impaired and diabetic glucose control. Nature 2013, 498:99-103.

17. Qin J, Li Y, Cai Z, Li S, Zhu J, Zhang F, Liang S, Zhang W, Guan Y, Shen D: A metagenome-wide association study of gut microbiota in type 2 diabetes. Nature 2012, 490:55-60.

18. Akhtar S, Nasir JA, Abbas T, Sarwar A: Diabetes in Pakistan: A systematic review and meta-analysis. Pakistan journal of medical sciences 2019, 35:1173.

19. Hume M, Sohail MU: Denaturing Gradient Gel Electrophoresis-Polymerase Chain Reaction Comparison of Chitosan Effects on Anaerobic Cultures of Broiler Cecal Bacteria and Salmonella Typhimurium. Foodborne pathogens and disease 2018, 15:246-252.

20. Sohail MU, Hume ME: Evaluation of Antimicrobial Action of Chitosan and Acetic Acid on Broiler Cecal Bacterial Profiles in Anaerobic Cultures Inoculated With Salmonella Typhimurium. The Journal of Applied Poultry Research 2019, 28:176-183.

21. Wajid B, Sohail MU, Ekti AR, Serpedin E: The A, C, G, and T of Genome Assembly. BioMed research international 2016, 2016.

22. Andrews S: FastQC: A quality control application for high throughput sequence data. Babraham Institute Project page: http://www bioinformatics bbsrc ac uk/projects/fastqc 2012. 
23. Callahan BJ, McMurdie PJ, Rosen MJ, Han AW, Johnson AJA, Holmes SP: DADA2: high-resolution sample inference from Illumina amplicon data. Nature methods 2016, 13:581.

24. Bokulich NA, Kaehler BD, Rideout JR, Dillon M, Bolyen E, Knight R, Huttley GA, Caporaso JG: Optimizing taxonomic classification of marker-gene amplicon sequences with QIIME 2's q2-featureclassifier plugin. Microbiome 2018, 6:90.

25. Hayden MR, Tyagi SC: $A^{\prime \prime}$ is for amylin and amyloid in type 2 diabetes mellitus. Jop 2001, 2:124-139.

26. Jha S, Sellin D, Seidel R, Winter R: Amyloidogenic propensities and conformational properties of ProlAPP and IAPP in the presence of lipid bilayer membranes. Journal of molecular biology 2009, 389:907-920.

27. Loomes KM: Survival of an islet $\beta$-cell in type-2 diabetes: curbing the effects of amyloid cytotoxicity. Islets 2011, 3:38-39.

28. Velasquez MT, Bhathena SJ, Hansen CT: Leptin and its relation to obesity and insulin in the SHR/Ncorpulent rat, a model of type II diabetes mellitus. Journal of Diabetes Research 2001, 2:217-223.

29. Wannamethee SG, Tchernova J, Whincup P, Lowe GD, Kelly A, Rumley A, Wallace AM, Sattar N: Plasma leptin: associations with metabolic, inflammatory and haemostatic risk factors for cardiovascular disease. Atherosclerosis 2007, 191:418-426.

30. Stumvoll M, Goldstein BJ, van Haeften TW: Pathogenesis of type 2 diabetes. In Type 2 Diabetes. CRC Press; 2016: 27-40

31. Bandaru P, Shankar A: Association between plasma leptin levels and diabetes mellitus. Metabolic syndrome and related disorders 2011, 9:19-23.

32. Maahs DM, Hamman RF, D'Agostino Jr R, Dolan LM, Imperatore G, Lawrence JM, Marcovina SM, Mayer-Davis EJ, Pihoker C, Dabelea D: The association between adiponectin/leptin ratio and diabetes type: the SEARCH for Diabetes in Youth Study. The Journal of pediatrics 2009, 155:133-135. e131.

33. Söderberg S, Zimmet P, Tuomilehto J, Chitson P, Gareeboo H, Alberti K, Shaw J: Leptin predicts the development of diabetes in Mauritian men, but not women: a population-based study. International journal of obesity 2007, 31:1126-1133.

34. Wannamethee SG, Lowe GD, Rumley A, Cherry L, Whincup PH, Sattar N: Adipokines and risk of type 2 diabetes in older men. Diabetes care 2007, 30:1200-1205.

35. Schmidt M, Duncan B, Vigo A, Pankow J, Couper D, Ballantyne C, Hoogeveen R, Heiss G, investigators A: Leptin and incident type 2 diabetes: risk or protection? Diabetologia 2006, 49:2086-2096.

36. Sun Q, van Dam RM, Meigs JB, Franco OH, Mantzoros CS, Hu FB: Leptin and soluble leptin receptor levels in plasma and risk of type 2 diabetes in US women: a prospective study. Diabetes 2010, 59:611-618.

37. Sohail MU, Althani A, Anwar H, Rizzi R, Marei HE: Role of the gastrointestinal tract microbiome in the pathophysiology of diabetes mellitus. Journal of diabetes research 2017, 2017. 
38. Long J, Cai Q, Steinwandel M, Hargreaves MK, Bordenstein SR, Blot WJ, Zheng W, Shu XO:

Association of oral microbiome with type 2 diabetes risk. Journal of periodontal research 2017, 52:636-643.

39. Shillitoe E, Weinstock R, Kim T, Simon H, Planer J, Noonan S, Cooney R: The oral microflora in obesity and type-2 diabetes. Journal of oral microbiology 2012, 4:19013.

40. Leiva-Gea I, Sánchez-Alcoholado L, Martín-Tejedor B, Castellano-Castillo D, Moreno-Indias I, UrdaCardona A, Tinahones FJ, Fernández-García JC, Queipo-Ortuño MI: Gut microbiota differs in composition and functionality between children with type 1 diabetes and MODY2 and healthy control subjects: a case-control study. Diabetes Care 2018, 41:2385-2395.

41. Stenman L, Waget A, Garret C, Klopp P, Burcelin R, Lahtinen S: Potential probiotic Bifidobacterium animalis ssp. lactis 420 prevents weight gain and glucose intolerance in diet-induced obese mice. Beneficial microbes 2014, 5:437-445.

42. Tong X, Xu J, Lian F, Yu X, Zhao Y, Xu L, Zhang M, Zhao X, Shen J, Wu S: Structural alteration of gut microbiota during the amelioration of human type 2 diabetes with hyperlipidemia by metformin and a traditional Chinese herbal formula: a multicenter, randomized, open label clinical trial. MBio 2018, 9:e02392-02317.

43. Neyrinck AM, Possemiers S, Verstraete W, De Backer F, Cani PD, Delzenne NM: Dietary modulation of clostridial cluster XIVa gut bacteria (Roseburia spp.) by chitin-glucan fiber improves host metabolic alterations induced by high-fat diet in mice. The Journal of nutritional biochemistry 2012, 23:51-59.

44. Nisar J, Mustafa I, Anwar H, Sohail MU, Hussain G, Ullah MI, Faisal MN, Bukhari SA, Basit A: Shiitake culinary-medicinal mushroom, Lentinus edodes (Agaricomycetes): a species with antioxidant, immunomodulatory, and hepatoprotective activities in hypercholesterolemic rats. International journal of medicinal mushrooms 2017, 19.

\section{Figures}



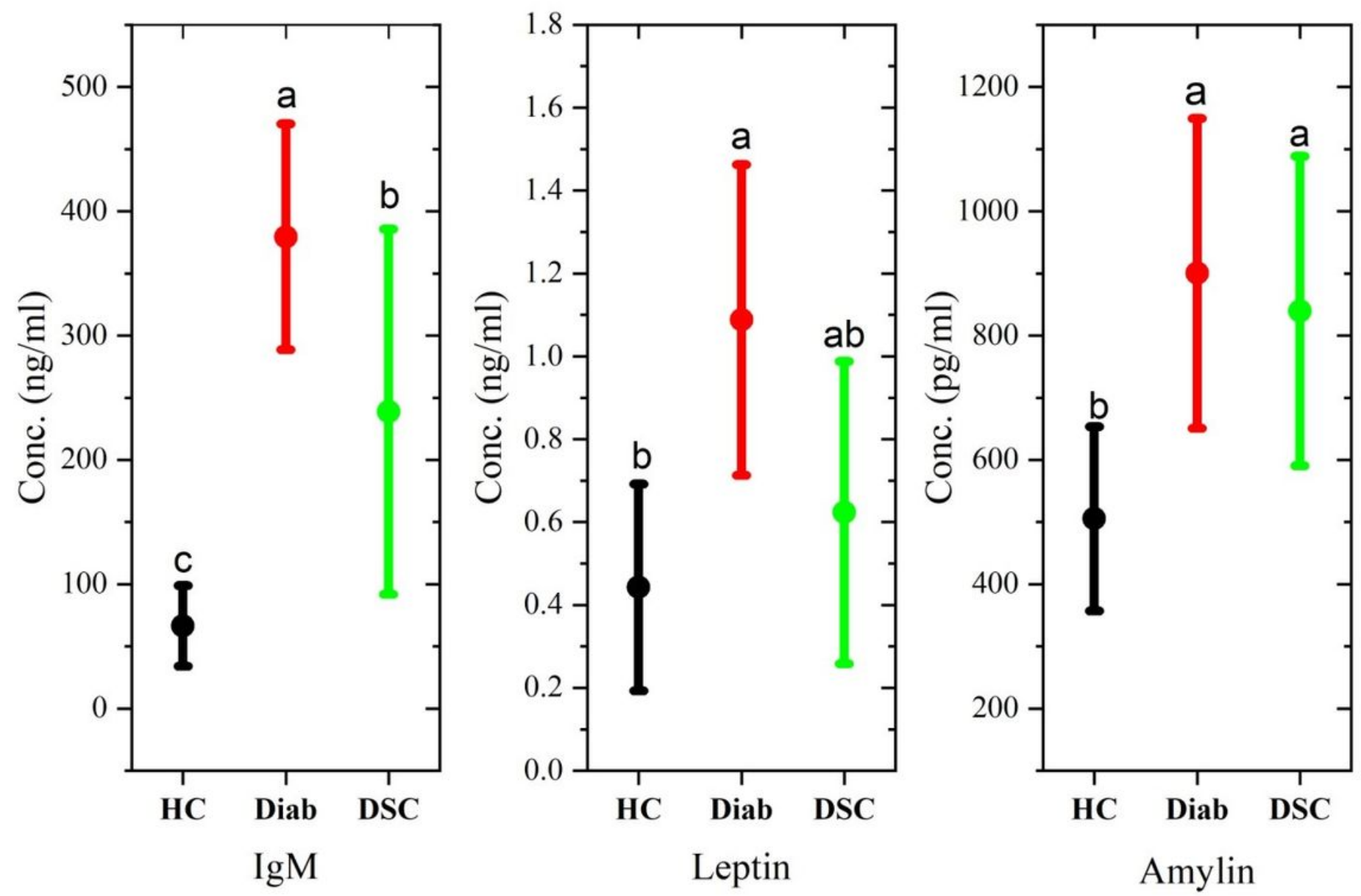

Figure 1

Serum concentrations of IgM, Leptin, and Amylin. Groups with different superscripts (a, b, and c) show that the serum levels of the markers differ significantly between the groups.
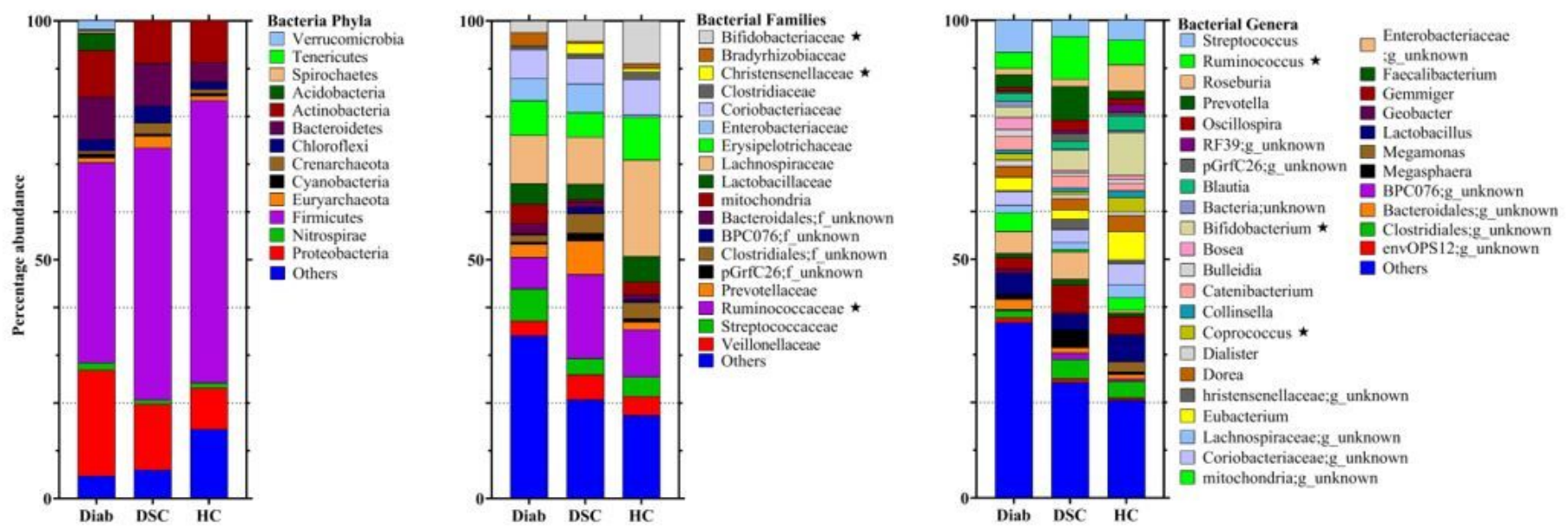

Figure 2 
Taxonomic distribution of the microbiome at the phylum, family and species level. Height of each bar represents the relative proportion of that phylotype. Only microbes with a relative proportion of at least $0.5 \%$ are presented in the bar plots. Taxon with asterisks differ significantly $(P \geq 0.05)$ between the study groups.
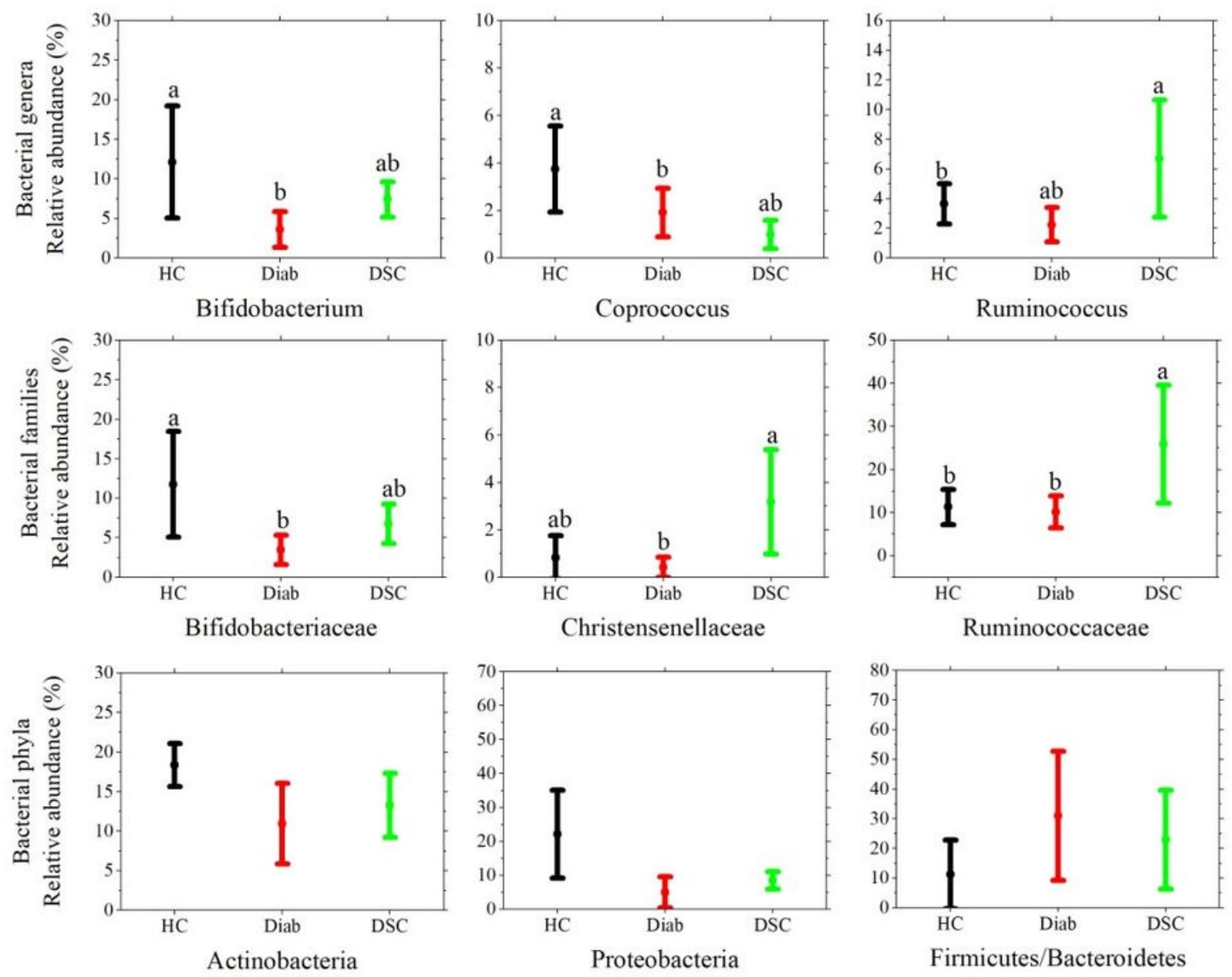

Figure 3

A box and whisker plot of the estimated means of the statistically different bacteria at phylum, family, and genus levels. Groups with different superscripts ( $a$ and $b$ ) show that the bacterial population differ significantly between the groups. 

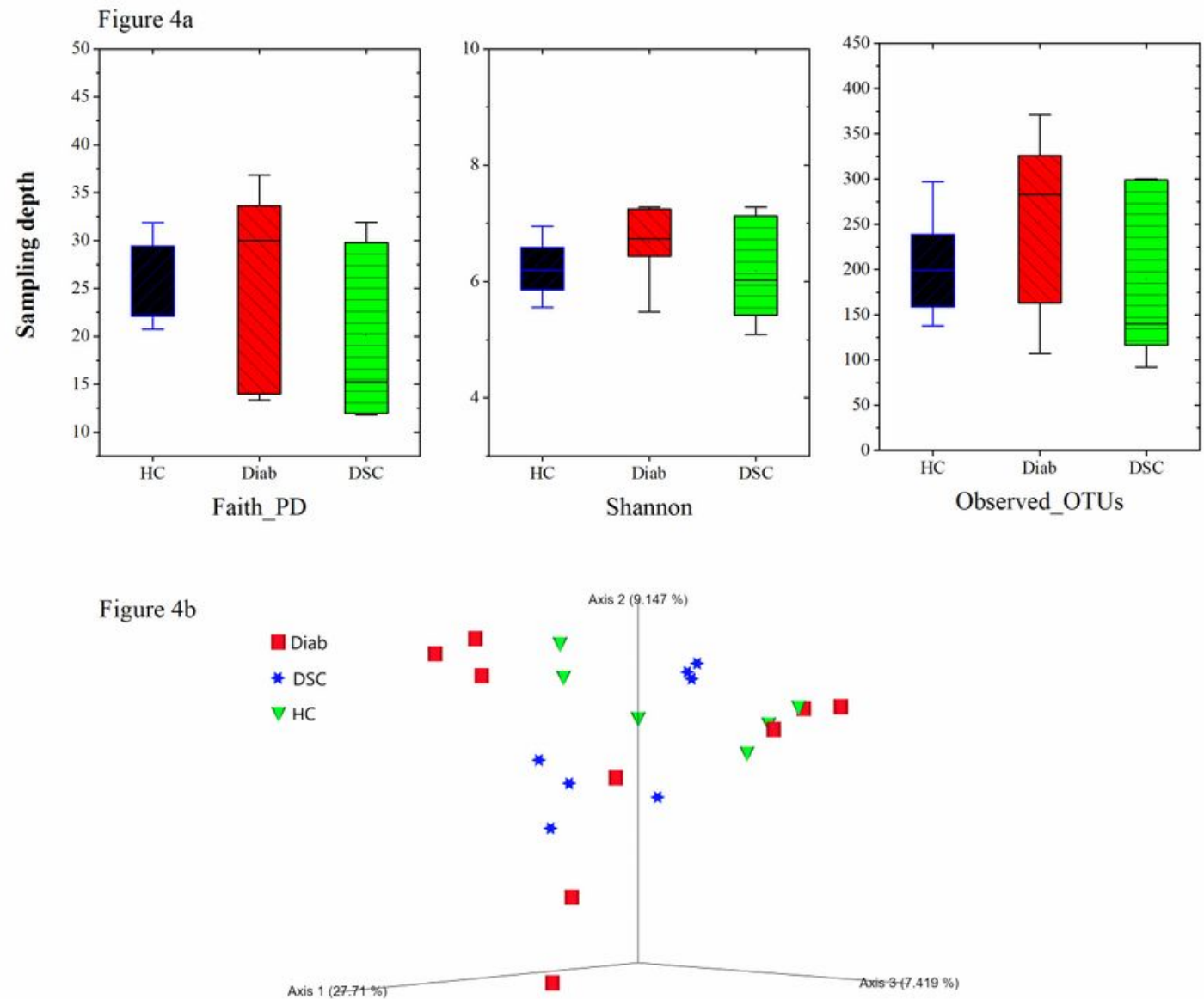

\section{Figure 4}

Alpha- and beta-diversity analysis at 15,653 sequencing depth. No statistical differences are observed between the study groups. 